\title{
Survival Analysis of Time to First Trimester induced Abortion of women in Harar Regional State, Ethiopia
}

Million Wesenu Demissie ( $\square$ Millionwesu@gmail.com )

Haramaya University

Kindu Kebede

Haramaya University

Research article

Keywords: Acceleration failure time; Abortion care; First trimester; survival time

Posted Date: April 30th, 2020

DOI: https://doi.org/10.21203/rs.3.rs-23485/v1

License: (c) (1) This work is licensed under a Creative Commons Attribution 4.0 International License.

Read Full License 


\section{Abstract}

Background: Abortion is the expulsion of a fetus from the uterus before it has reached the stage of viability. Abortion among adolescents and youth is a major public health issue, especially in developing countries like Ethiopia. Therefore, the present study was headings to the survival time of first trimester women abortion and associated factors among women whose age group in 15-49 years admitted to Jugel Hospital in Harar city, Ethiopia.

Method: A hospital record pregnant women were reviewed and retrospective study design was conducted at Jugel Hospital in Harar regional state from January 2018 to December 2020.The study was conducted among nine hundred and ninety seven sampled women with abortion. The data was entered into Stata version-14 for cleaning and data analysis. Cox proportional hazard and multivariate accelerated failure time regression with log-logistic distribution were carried out to determine significant association between response variable and covariates at $5 \%$ level statistical significance was ascertained at $5 \%$ level of significance.

Result: The result from log-rank estimation revealed that the survival of a woman significantly associated with age of mother, gravidity, parity, abortion care, place of managed and type of uterine evacuation procedure of mother. Based on AIC and BIC criteria and.The log-logistic of acceleration failure time model has small AIC and BIC among compared models. The multivariate log-logistic acceleration failure time model result indicated that age of mother being 30-34years $(\Phi=1.1116,95 \% \mathrm{Cl}=(1.0144,1.2181))$, being in age group $>=35$ years $(\Phi=1.1607,95 \% \mathrm{Cl}=(1.0388,1.2969))$ having gravida $3-4(\Phi=0.9442,95 \% \mathrm{Cl}=$ $(0.8960,0.9951))$, safe abortion care $(\Phi=0.7805,95 \% \mathrm{Cl}=(0.7156,0.8513))$, in-patient place of managed $(\Phi=1.1582,95 \% \mathrm{Cl}=(1.0628,1.2621))$ and type of uterine evacuation of $\mathrm{MVA}(\Phi=1.0636,95 \% \mathrm{Cl}=$ $(1.0125,1.1174))$ was covariates significantly associated to survival time women abortion in first trimester stage.

Conclusions: The findings of this study showed that age of mother, gravidity, abortion care, place of managed and type of uterine evacuation for abortion were potential covariates associated to survival time of women abortion. It is recommended that women should be aware on the burden of those risk factors and health workers should be give due attention to this proximate determinants on abortion case.

\section{Background}

Abortion is the medical term for any pregnancy loss before the fetus is viable. The World Health Organization (WHO) defined unsafe abortion as a procedure for terminating a pregnancy performed by persons lacking the necessary skills or in an environment, not in conformity with minimal medical standards, or both[1]. Globally, it is estimated that 47000 women die each year from complications related to unsafe abortion. Many of these deaths could be prevented through better access to sexual education, contraceptive information and supplies, and safe abortion services where allowed by law [2]. About $97 \%$ of all global abortions between 2010 and 2014 occurred in low income countries[3]. Abortion 
related complications were the most burdened health problem and the negative effects of unsafe abortions are disproportionately higher in Africa as study conducted by[4]. Study conducted in Mozambique suggested that low degree of autonomy for women, the limited availability of health facilities providing abortion services and a lack of patient-centeredness of health services were potential determinants of abortion [5]. Induced abortions occurred in the world, almost half of which were unsafe abortion and a huge public health problem worldwide, particularly in developing countries. In East Africa, the estimated abortion rate in 2003 was 39 per 1,000 women aged 15-44 years, in central and west Africa, the rate was 26-28 per 1,000 women aged 15-44; all of the procedures in these sub-regions were judged to be unsafe [6]. The study done by[7] in Kurdistan Region of Iraq concluded that age of mother, number of children, level of education and economic status of women have significant association with abortion of women using binary logistic regression models.

In Ethiopia, a cross-sectional study conducted October 2011 - April 2012 in Jimma town that considered 808 safe abortion cases and 21 unsafe abortion cases. Women with the age group 20-24 years' experience about $46 \%$ first trimester abortion and $27 \%$ second trimester abortion [8]. According to study conducted by [3], the current problem of Ethiopia is unwanted pregnancy which estimated that out of 4 million pregnancies about $42 \%$ were unwanted pregnancy leading to 382000 induced abortion. In fact, the Ethiopian abortion law is the result of several years' effort by a coalition of health and women's rights advocates who have worked together to ensure women better access to safe abortion. However, a liberal abortion law in a country does not guarantee access to safe abortion services. The revised law experienced in Ethiopia government before in 2005, invited a woman to get safe and legal abortion when the woman pregnant as a result of rape, has disability in mentally or physically, her age is unprepared for childbirth and if proceeding with pregnancy put her physically health at risk[9].

In developing country like Ethiopia, first trimester abortion is critically very important where the health facility is unbalanced with abortion case of late gestational age. The knowledge of health providers about the abortion care was low to ensure appropriate treatment and prompt referral for immediate complications of unsafe or incomplete abortion and health facilities were not well equipped infrastructure [10].

As stated in literature, many studies conducted in lined to abortion related in Ethiopia that mainly focused on the knowledge of health providers about abortion [10], patient satisfaction on service[11],the reason for abortion and unmet need for family planning[12, 13], the expansion of abortion services[14] and prevalence of induced abortion [15]. In spite of the fact that, several studies have been done on assessment and examined general abortion care, they didn't deal with survival time of first trimester abortion gestational age. The current study aims to survival time of women with first trimester abortion gestational age and its associated factor with that of first trimester abortion since there is limited information about this critical time of women pregnancy and unsafe abortion. In order fill the gap, the study was used survival models of cox proportional hazard and acceleration failure time that predict survival time of each factor with first trimester abortion of women . 


\section{Methods}

\section{The Study Area, Design and Period}

The study was conducted in Harar town which found in Eastern Ethiopia at Harar regional state in Ethiopia. The town is found $526 \mathrm{~km}$ away from Addis Ababa which is capital city of Ethiopia. The crosssectional study conducted during January 1, 2018 to December 30, 2020 in which medical records of 997 of women with abortion were reviewed.

\section{Study Population}

All women who came for complication of abortion and took treatment services in Jugel Hospital, Harar town, Ethiopia were considered. The target population was recorded patients of laboratory confirmed abortion of women. The survival data were extracted from the comprehensive abortion care service chart which contained clinical information.

\section{Variables Included in this Study}

The response/outcome variable is the survival time of trimester abortion in women, the length of time from pregnancy date until the date of first trimester abortion of women measured in weeks. The status variable is coded as 1 for first trimester of abortion and 0 censored for not first trimester abortion. The predictor variables in survival data analysis are called covariates. These covariates can be categorical, count or continuous. The predictor variables or factors which are assumed to influence the survival time of abortion for women. The covariates that can be included in this study are listed below.

Table 1: Description of covariates that is included in this study

\begin{tabular}{l|l}
\hline Variables & Alternative choice $/$ labels \\
\hline Age of mother & $0=15-19,1=20-24,2=25-29,3=30-34,4=>=35$ \\
Gravidity & $0=1-2,1=3-4,2=>=5$ \\
Para & $0=$ None, $1=1-2$ Parity, $2=>=3$ Parity \\
Abortion care & $0=$ Post, $1=$ Safe \\
Post abortion contraception & $0=$ Counseled, $1=$ New acceptor, $2=$ Repeat acceptor \\
Contraceptive method & $0=$ No, 1=Yes \\
Type of uterine evacuation & $0=$ MA, $1=$ MVA, $2=$ Both MA and MVA \\
Place of managed abortion & $0=$ Out-patient 1=In-patient \\
\hline
\end{tabular}

\section{Method of Data Analysis}

The data was analyzed using Stata-14 version. To summarize the data descriptive statistics such as frequency, percentages for categorical data and non-parametric test like log-rank that test equality of survivor functions between covariate levels were used. A univariate cox proportional hazard regression was used to select potential candidate covariates for multivariate cox proportional hazard regression model. During analysis of univariate cox proportional hazard regression, a significance of less than $10 \%$ was used as a cut of point. Schoenfeld residuals and log-log plot of survival or graphically assess proportional-hazard assumption were used to check proportionality assumption between levels of 
categorical variables which was significant in univariate proportion hazard regression before fitting final model. The violation of proportional hazard assumption leads the analysis to parametric accelerated failure time model[16]. To specify the appropriate base line distribution from exponential, weibull, loglogistic and log-normal distribution, the model comparisons have been done using Akaike information criteria (AIC) and Bayesian information criteria (BIC). A model that have minimum AIC and BIC was selected as appropriate parametric accelerated failure time to describe the data well. Multivariate accelerated failure time model was used to predict survival time and identify covariates that associated to survival time first trimester of women abortion in Harar, Ethiopia. Wald test, p-value, time ratio and confidence interval of time ratio respectively were used identify the relationship between potential covariates and first trimester of women abortion. The statistical significance of multivariate accelerated failure time of final model with specific baseline distribution was explicitly stated by $p$-value and confidence interval. An acceleration factor (time ratio) was used to announce the rate of increment or decrement of survival time for women abortion as compared to reference category. The model fitness was checked by likelihood ratio test and Pseudo $\mathrm{R}^{2}$ in addition to graphical technique.

\section{Results}

\section{Proportion of associated factors to induced first trimester abortion of women}

A total of 997 women with abortion at Jugel Hospital were considered. After the medical cards of women were reviewed among those abortion of women of 808 (81.04\%) first trimester and the remaining 189 (18.96\%) were censored. Based on the summary statistics in Table 2 below, the first trimester percentages of abortion of women, $16.75 \%, 27.68 \%, 22.87 \%, 9.13 \%$ and $4.61 \%$ were in the age group of mother $15-19,20-24,25-29,30-34$ and greater than or equal to 35 , respectively.

The proportion for number of pregnancy for women who had 1-2 pregnancy was $54.96 \%$ (548) in first trimester abortion time which is more than the women who had 3-4 and greater than or equal to five number of pregnancy that was $19.56 \%(195)$ and $6.52 \%(65)$ respectively. Of the total $39.02 \%(389)$ women had one up-to two number of parity in the first trimester whereas $39.32 \%$ (392) had none parity and $2.71 \%(27)$ had parity greater than or equal to three in the first trimester abortion. Abortion care of mother is a very critical factor for survival of mother during pregnancy, in this study about $53.16 \%(530)$ women have safe abortion care in Harar town and the remaining $46.84 \%$ have post abortion care which needs improve to survive more mother in the reproductive age. Due attention should be given to women's' place of managed abortion since most women took treatment out of health center that is $50.95 \%$ ( 508) out of total 997 women under study in which about $47.84 \%$ (477) found in the first trimester abortion gestational age. Some comparable proportion of women had treatment in health center, specifically $33.20 \%$ (331) that was in first trimester abortion gestational age. Most of the women considered/participated under this study were found in age group 20-24 years, have number of pregnancy 1-2, safe abortion care, manual vacuum aspiration uterine evacuation, new acceptor of post abortion contraception, and users of contraceptive methods which have frequency of $331(33.20 \%), 667$ 
(66.9\%), 530(53.16\%), $658(66.00 \%), 518(51.96 \%)$ and 838(84.05\%) respectively as found Table 2 in detail.

Table 2: Summary of Descriptive Statistics for covariates that included in this Study

\begin{tabular}{|c|c|c|c|}
\hline \multicolumn{4}{|c|}{ Response variable (Status of abortion) } \\
\hline censored & count $\%$ first trir & mester count $\%$ & Total count $\%$ \\
\hline \multirow[t]{5}{*}{ Age of mother } & $\begin{array}{c}15-19 \\
\end{array}$ & 242.41 & 16716.7519119 .16 \\
\hline & $20-24$ & $55 \quad 5.52$ & 27627.6833133 .20 \\
\hline & $25-29$ & 494.91 & 22822.8727727 .78 \\
\hline & $30-34$ & $37 \quad 3.71$ & $919.13 \quad 12812.84$ \\
\hline & $>=35$ & $24 \quad 2.41$ & $464.61 \quad 70 \quad 7.02$ \\
\hline \multirow[t]{3}{*}{ Number of pregnancy for women/gravida } & $1-2$ & 11911.94 & 54854.9666766 .90 \\
\hline & $3-4$ & $43 \quad 4.31$ & 19519.5623823 .87 \\
\hline & $>=5$ & $27 \quad 2.71$ & 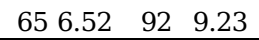 \\
\hline \multirow[t]{3}{*}{$\overline{\text { Para }}$} & None & 696.92 & 39239.3246146 .24 \\
\hline & 1-2 parity & 10310.33 & 38939.0249249 .35 \\
\hline & $>=3$ parity & 171.71 & $272.71 \quad 44 \quad 4.42$ \\
\hline \multirow[t]{2}{*}{ Abortion care } & Post & 15315.35 & 31431.4946746 .84 \\
\hline & Safe & 363.61 & 49449.5553053 .16 \\
\hline \multirow[t]{2}{*}{ Place of managed } & Outpatient & 313.11 & 47747.8450850 .95 \\
\hline & Inpatient & 15815.85 & 33133.2048949 .05 \\
\hline \multirow[t]{3}{*}{ Type of uterine evacuation } & MA & 585.82 & 23223.2729029 .09 \\
\hline & MVA & 12712.74 & 53153.2665866 .00 \\
\hline & Both MA \&MVA & $\begin{array}{ll}4 & 0.40\end{array}$ & 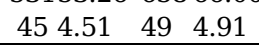 \\
\hline \multirow[t]{3}{*}{ Post-abortion Contraception } & Counseled & $54 \quad 5.42$ & 17617.6523023 .07 \\
\hline & New acceptor & 939.33 & 42542.6351851 .96 \\
\hline & Repeat acceptor & $42 \quad 4.21$ & 20720.7624924 .97 \\
\hline \multirow[t]{2}{*}{$\overline{\text { Contraceptive methods }}$} & No & 363.61 & 12312.3415915 .95 \\
\hline & Yes & 15315.35 & 68568.7183884 .05 \\
\hline
\end{tabular}

\section{Comparison of survival experience of first trimester abortion for women}

Survival time distributions for time-to-first trimester is estimated for each group using the K-M method and in order to compare the survival curves of two or more groups, log-rank test has been employed. The log-rank revealed that there is significant difference between survival experience abortion for women in predictor groups of age of mother, gravida, para, abortion care and place of managed abortion at $5 \%$ level of significance. The Kaplan-Meier survival plot of age group of mother also indicated that there is survival difference between age group of mother which implies that mother whose age group is greater than or equal to 35 years had higher survival time than others age groups of mother. The women having $>=5$ gravida had higher survival time than women having gravida 1-2 and 3-4 in experiencing first trimester abortion of women as indicated in Figure 1.

Table 3: log rank test for survival of each covariate 


\begin{tabular}{lcll} 
Explanatory Variables & DF & Chi2 value & p-value \\
\hline Age of mother & 4 & 24.7300 & 0.0001 \\
\hline Gravida & 2 & 6.24 & 0.0441 \\
\hline Para & 2 & 22.59 & 0.0001 \\
\hline Abortion care & 1 & 273.71 & 0.0001 \\
\hline Place of managed & 1 & 250.92 & 0.0001 \\
\hline Post abortion contraception & 2 & 2.68 & 0.2613 \\
\hline Contraceptive methods & 1 & 1.10 & 0.2936 \\
\hline
\end{tabular}

Comparing the survival function between abortion care of mother, the Kaplan-Meier estimates for two groups showed that women who had post abortion care had higher survival probability than those who had safe abortion care of first trimester. In addition, women treated in-patient had higher survival probability than out-patient in experiencing first trimester abortion and this confirmed the log-rank test result that stated there is significant difference between in-patient and out-patient survival of women.

\section{Univariate Survival Cox PH Analysis}

Single covariate Cox proportional hazards model analysis is an appropriate procedure that is used to screen out potentially important variables before directly included in the multivariate model. The relationship between each covariates and survival time of abortion for women are presented in Table 4 below. The result showed that age of mother, gravida, Para, abortion care, place of managed and type of uterine evacuation were significantly related to survival time of women abortion at $10 \%$ level of significance which is candidate predictors that were included in the final model.

Table 4: Univariate analyses of cox regression result for survival time of women abortion data set

\begin{tabular}{|c|c|c|c|c|c|c|}
\hline ovariates /levels & B & SE & $\mathrm{Z}$ & $\mathrm{p}$-value & HR & $90 \%$ CI for HR \\
\hline ye of mother $20-24$ & -0.0931 & 0.0912 & -1.0200 & 0.3080 & 0.9111 & {$[0.7842,1.0586]$} \\
\hline $25-29$ & -0.1545 & 0.0942 & -1.6400 & 0.1010 & 0.8568 & {$[0.7339,1.0004]$} \\
\hline $30-34$ & -0.3718 & 0.1147 & -3.2400 & 0.0010 & 0.6895 & {$[0.5709,0.8326]^{* *}$} \\
\hline$>=35$ & -0.4841 & 0.1408 & -3.4400 & 0.0010 & 0.6162 & {$[0.4888,0.7768]^{* *}$} \\
\hline ravida & 0.0675 & 0.0756 & 0.8900 & 0.3720 & 1.0698 & {$[0.9447,1.2115]$} \\
\hline$>=5$ & -0.2036 & 0.1115 & -1.8300 & 0.0680 & 0.8158 & {$[0.6791,0.9799]^{*}$} \\
\hline \multirow[b]{2}{*}{$>=3$} & -0.1863 & 0.0651 & -2.8600 & 0.0040 & 0.8300 & {$[0.7457,0.9239]^{* *}$} \\
\hline & -0.5839 & 0.1637 & -3.5700 & 0.0001 & 0.5577 & {$[0.4260,0.7302]^{* *}$} \\
\hline Jortion care safe & 0.9670 & 0.0670 & 14.4300 & 0.0001 & 2.6300 & {$[2.3555,2.9365]^{* *}$} \\
\hline ace of managed in-patient & -0.9212 & 0.0666 & -13.8300 & 0.0001 & 0.3980 & {$[0.3567,0.4441]^{* *}$} \\
\hline Ipe of uterine Ev. MVA & -0.2420 & 0.0714 & -3.3900 & 0.0010 & 0.7850 & {$[0.6380,0.8829]^{* *}$} \\
\hline Both MA\&MVA & 0.3369 & 0.1555 & 2.1700 & 0.0300 & 1.4005 & {$[1.0845,1.8087]^{* *}$} \\
\hline \multicolumn{7}{|l|}{ st abortion contraceptive } \\
\hline new acceptor & 0.1157 & 0.0796 & 1.4500 & 0.1460 & 1.1226 & {$[0.9849,1.2797]$} \\
\hline repeat acceptor & 0.0830 & 0.0918 & 0.9000 & 0.3660 & 1.0865 & {$[0.9342,1.2637]$} \\
\hline ontraceptive methods Yes & 0.0806 & 0.0867 & 0.9300 & 0.3520 & 1.0840 & [0.9400.1.2502] \\
\hline
\end{tabular}


$*, * * \& * * *$ shows the significances of Hazard Ratio (HR) at 0.05, 0.01 and 0.001 respectively.

Goodness of fit testing approach is appealing because it provides a test statistic and p-value for assessing the $\mathrm{PH}$ assumption for a given predictor of interest and rho is a relation between time and residuals. The result in (Table 5) showed that the test of correlation (rho) is significant for place of managed abortion and type of uterine evacuation covariates at $5 \%$ level of significance that indicates proportional hazards assumption is violated. Moreover, it is also possible see its global test which suggested that in this study assumptions of PH do not satisfied by the covariate in the model. Likewise, the scatter plots of Scaled Schoenfeld residuals in (Figure 3) below also used to check PH assumption. The plot for type of uterine evacuation against survival time was also slightly upward (not horizontal). These revealed that there is a violation of the proportional hazard assumption for the covariate type of uterine evacuation. Thus, we doubt the accuracy of the PH assumption and consider the AFT model for this data set.

Table 5: Test of proportional hazards assumption

\begin{tabular}{l|llll} 
Covariates & Rho & DF & Chi2 value & p-value \\
\hline Age & 0.024 & 1 & 0.54 & 0.4613 \\
Gravida & -0.029 & 1 & 0.86 & 0.3531 \\
Para & 0.024 & 1 & 0.51 & 0.4742 \\
Abortion care & -0.047 & 1 & 2.07 & 0.1502 \\
Place of managed & 0.069 & 1 & 4.66 & 0.0308 \\
Type of uterine evacuation & 0.126 & 1 & 17.47 & 0.0000 \\
\hline \multicolumn{1}{c}{ Global test } & & 6 & 62.24 & 0.0000 \\
\hline
\end{tabular}

Chi2 $=$ chi-squared, $\mathrm{DF}=$ degree of freedom

\section{Accelerated Failure Time Model}

To fit the AFT model we have applied four parametric survival models (exponential, weibull, lognormal and log-logistic) to the effect of covariates to first trimester abortion of women under this study. The AIC and BIC value of log-logistic AFT model was the minimum among all other AIC and BIC values of the models suggesting that it was the most appropriate model to describe the abortion of women dataset.

Table 6: Comparison of AFT models using AIC and BIC criteria for abortion of women data 


\begin{tabular}{lllll}
\hline Baseline distribution & Null model log likelihood & $\begin{array}{l}\text { Full model } \\
\text { Log likelihood }\end{array}$ & AIC & BIC \\
\hline Exponential & -1135.603 & -1080.988 & 2187.976 & 2251.738 \\
\hline Weibull & -838.6372 & -673.7411 & 1375.482 & 1444.149 \\
\hline Log-logistic & -660.0195 & -487.1832 & 1002.366 & 1071.033 \\
\hline Lognormal & -678.7945 & -520.6901 & 1069.380 & 1138.047 \\
\hline
\end{tabular}

\section{Presentation of final AFT model and interpretation of parameters}

The output of final log-logistic model was presented in Table 7 below. Thus, the result showed that age of mother, gravida, abortion care, place of managed and type of uterine evacuation were significantly associated to abortion of women. From this output, women with age group of 30-34 \& >=35, place of managed abortion in hospital (in-patient) and MVA type of uterine evacuation procedure for mother statistically significant and prolonged survival time of women with abortion while having number of pregnancy 3-4 and safe abortion care of mother shorten survival time of women abortion. When the effect of other covariate held constant, the estimated acceleration factor for mother whose age group in $30-34 \&>=35$ years is estimated to be 1.1116 with [95\% Cl: $1.0144,1.2181]$ and 1.1607 with [95\% Cl: $1.0388,1.2969]$ respectively. The confidence interval for the acceleration factor did not include one and $\mathrm{P}$ value is small ( $p=0.0234 \& 0.0085)$. This indicates women with age group in $30-34 \&>=35$ years have longer survival time than the age group of 15-19 women. But the age groups 20-24 \& 25-29 of women were not significantly different from the age group 15-19 at 5\% level of significance. The estimated acceleration factor for women having number of pregnancy 3-4 was estimated to be 0.9442 with $95 \%$ confidence interval $[0.8960,0.9951]$. This indicates that women who had gravida 3-4 have shorten survival time than those who had gravida 1-2. This implied that women who had gravida 1-2 survived $5.58 \%$ longer than women who had gravida $3-4$.

Similarly the acceleration factor for women who had safe abortion care was estimated to be 0.7805 with $95 \%$ confidence interval [0.7156, 0.8513]. The interval did not include base line comparison and $p$-value is very small $(p=0.0001)$ that indicates the women who had post abortion care prolonged survival time than women who had safe abortion care. This means, the women who had post abortion have survived $21.95 \%$ times longer than those who had safe abortion care. In addition, the estimated acceleration factor for women treated at hospital (in-patient) was estimated to be 1.1582 with $95 \%$ confidence interval $[1.0628,1.2621]$ and the corresponding $p$-value is very small $(p=0.0008)$. This shows that women who have treatment at hospital have survived $15.82 \%$ times longer than women who have treatment out of hospital.

Table 7: The summary of Final log-logistic Accelerated Failure Time (AFT) Model 


\begin{tabular}{|c|c|c|c|c|c|c|}
\hline Covariate & $\mathrm{B}$ & $\mathrm{SE}$ & Wald & Sign. & $\Phi$ & $95 \%$ CI for $\Phi$ \\
\hline Age $\quad \operatorname{Ref}(15-19)$ & & & & & & \\
\hline $20-24$ & 0.0447 & 0.0299 & 1.496 & 0.1346 & 1.0458 & {$[0.9862,1.1089]$} \\
\hline $25-29$ & 0.0284 & 0.0391 & 0.725 & 0.4686 & 1.0288 & {$[0.9528,1.1108]$} \\
\hline $30-34$ & 0.1058 & 0.0467 & 2.267 & 0.0234 & 1.1116 & {$[1.0144,1.2181]^{*}$} \\
\hline$>=35$ & 0.1490 & 0.0566 & 2.633 & 0.0085 & 1.1607 & {$[1.0388,1.2969]^{*}$} \\
\hline $\operatorname{Ref}(1-2)$ & & & & & & \\
\hline $3-4$ & -0.0574 & 0.0268 & -2.142 & 0.0322 & 0.9442 & {$[0.8960,0.9951]^{*}$} \\
\hline$>=5$ & -0.0713 & 0.0472 & -1.511 & 0.1308 & 0.9312 & {$[0.8489,1.0214]$} \\
\hline \multirow{4}{*}{$\begin{array}{ll}\text { Para } & \text { Ref(none) } \\
& 1-2 \text { parity } \\
& >=3 \text { parity } \\
\text { Abortion care Ref(post) }\end{array}$} & & & & & & \\
\hline & 0.0418 & 0.0323 & 1.293 & 0.1960 & 1.0427 & {$[0.9787,1.1109]$} \\
\hline & 0.0825 & 0.0763 & 1.081 & 0.2797 & 1.0860 & {$[0.9351,1.2612]$} \\
\hline & & & & & & \\
\hline Safe & -0.2478 & 0.0443 & -5.592 & 0.0001 & 0.7805 & {$[0.7156,0.8513]^{* * *}$} \\
\hline \multirow{2}{*}{$\begin{array}{ll}\text { Place of managed } & \begin{array}{l}\text { Ref(outpatient) } \\
\text { Inpatient }\end{array}\end{array}$} & & & & & & \\
\hline & 0.1468 & 0.0438 & 3.349 & 0.0008 & 1.1582 & {$[1.0628,1.2621]^{* *}$} \\
\hline Type of uterine Ref(MA) & & & & & & \\
\hline & 0.0617 & 0.0252 & 2.452 & 0.0142 & 1.0636 & {$[1.0125,1.1174]^{* *}$} \\
\hline Both MA \& MVA & -0.0262 & 0.0523 & -0.501 & 0.6161 & 0.9741 & {$[0.8793,1.0792]$} \\
\hline Constant & 2.2264 & 0.0530 & 42.026 & 0.0001 & 9.2661 & {$[8.3523,10.2800]^{* * *}$} \\
\hline$\overline{L n(g a m m a)}$ & -1.6323 & 0.0301 & -54.259 & 0.0001 & & {$[-1.6912,-1.5733]$} \\
\hline Gamma & 0.1955 & 0.0059 & & & & {$[0.1843,0.2074]$} \\
\hline
\end{tabular}

Ref : stand's for reference categories, MA=manual aspiration, MVA=manual vacuum aspiration

$*$, ** $\& * * *$ shows the significances of time ratio $(\Phi)$ at $0.05,0.01$ and 0.001 respectively

The plot for the log-logistic baseline distribution approximately makes straight line better than exponential, weibull and log-normal baseline distribution. This evidence also strengthens the decision made by AIC value that log-logistic baseline distribution is appropriate for the given dataset as observed in Figure 4.

The likelihood ratio test for log-logistic in Table 8 ,shows that the model is significant and the log likelihood values of the null model and the full model indicates that the model has a significant improvement after the covariates were added in the model. In addition, the model fitted in this study have $R^{2}$ statistic value of 0.1592 implying a good fit of the model since a perfectly adequate model has low $R^{2}$ due to high percent of censored data. Thus, all in all we can say that our model fits the data very well.

Table 8: Likelihood ratio test of model adequacy

\begin{tabular}{llcccc} 
Log-likelihood (null model) & Log-likelihood(full model) & Chi-sq & DF & P-value & $R^{2}$ \\
\hline-660.01954 & -487.18321 & 345.67 & 12 & 0.0001 & 0.1592
\end{tabular}

\section{Discussion}

This study show that log-logistic AFT model was most appropriate model which has small AIC and BIC as compared with other parametric model and the data set were good fit to describe the abortion of women data sets (refer to Table 6\&8). Thus, the result showed that age of mother, gravida, abortion care, place of managed for abortion and type of uterine evacuation of abortion were significantly associated to survival time of first trimester abortion of women(refer to Table 7). From this output, women with age group of $30-34 \&>=35$ had longer survival time than those whose age group of $15-19$. This finding is 
consistent with the study by [7-9]. Women that had post abortion care after completed abortion were more prolonged survival time than those who had safe abortion care. This had been confirmed by the study $[2,4,10]$. Moreover, women who treated abortion at hospital had more survived than those who treated out of hospital. This result is in line with the previous study $[10,14]$. However, women who had gravida 3-4 had shortened survival time than those who had gravida 1-2. This result is linked with the previous study by [7]. Women who used manual vacuum aspiration type of uterine evacuation procedure had longer prolonged survival time of women as compared those who used manual aspiration type of uterine evacuation.

\section{Conclusion}

The main purpose of this study was identifying the potential covariates that associated to the survival probability of abortion for woman in Harar regional state. Therefore, the result of log-logistic acceleration failure time model implies age of mother, gravidity, abortion care, place of managed abortion and type of uterine evacuation of mother were significant predictors for survival time of women abortion at a $5 \%$ level of significance. Women who belongs to age group 30-34 \& $>=35$ had more survived than those who had age group 15-19. Furthermore, women who had gravida 1-2 were more survived than those who had more than 2 gravida. Likewise, a woman who holds by post abortion care had more survived than those who had safe abortion care and women who take treatment at hospital had more survived than those who take treatment out of hospital. Hence, special care should be given to the pregnant women during at first trimester gestational age to survive from complications of abortion and midwifery should be intervening critically to use family planning which reduces gravida or number of pregnancy.

\section{Abbreviations}

$\mathrm{AFT}=$ Accelerated failure time, $\mathrm{AIC}=$ Akaike information criteria, $\mathrm{BIC}=$ Bayesian information criteria, $\mathrm{Cl}=$ Confidence interval, $\mathrm{DF}=$ Degrees of freedom, $\mathrm{KM}=$ Kaplan-Meier, $\mathrm{MA}=$ manual Aspiration, $\mathrm{MVA}=$ manual vacuum aspiration, $\mathrm{PH}=$ Proportional hazard, $\mathrm{PO}=$ Proportional odds, $\mathrm{WHO}=$ World health organization, $\mathrm{HR}=$ Hazard Ratio,SE = Standard Error, Chi2=chi-squared.

\section{Declarations}

\section{Ethics approval and consent to participate}

Ethical approval was obtained from Haramaya University College of Computing and Informatics, and Research and Ethical committee. The data was collected through a review of medical records using a data collection sheet prepared by the researcher. The names of women will not be used on the data collection form.

\section{Consent for publication}

Not applicable. 


\section{Availability of data and material}

The raw data used in this study can be accessed from Jugel Hospital patient charts. The data in our study will not be shared in order to protect the participants' anonymity.

\section{Competing interests}

The authors declare that they have no competing interests.

\section{Funding}

None.

\section{Authors' contributions}

MW and KK have made substantial contribution to conception, design, analysis and interpretation of data. We involved in drafting the manuscript, revising it critically for important intellectual content. All authors have read and approved the manuscript.

\section{Acknowledgments}

Jugel Hospital Director and all staff are gratefully acknowledged for the data they supplied for this health research.

\section{References}

1. Ganatra B, et al. From concept to measurement: operationalizing WHO's definition of unsafe abortion. Bull World Health Organ. 2014;92(3):155.

2. Nations U, Department of Economic and Social Affairs; Population Division. World Population Ageing 2015. 2013, Author New York, NY.

3. Ganatra B, et al. Global, regional, and subregional classification of abortions by safety, 2010-14: estimates from a Bayesian hierarchical model. The Lancet. 2017;390(10110):2372-81.

4. Klutsey EE, Ankomah A. Factors associated with induced abortion at selected hospitals in the Volta Region, Ghana. International Journal of Women's Health. 2014;6:809.

5. Frederico $\mathrm{M}$, et al. Factors influencing abortion decision-making processes among young women. Int J Environ Res Public Health. 2018;15(2):329.

6. Organization WH, Unsafe abortion: global and regional estimates of incidence of unsafe abortion and associated mortality in 2003.. 2007, World Health Organization.

7. AKBAY CA, Azo FM. Prevalence and risk factors of abortion among a sample of married women in Kurdistan Region of Iraq. Zanco Journal of Medical Sciences. 2016;20(3):1424-32.

8. Bonnen KI, Tuijje DN, Rasch V. Determinants of first and second trimester induced abortion-results from a cross-sectional study taken place 7 years after abortion law revisions in Ethiopia. BMC 
Pregnancy Childbirth. 2014;14(1):416.

9. Negasso G. The Revised Family Code. 2000.

10. Taddele T, et al. Factors associated with health care provider knowledge on abortion care in Ethiopia, a further analysis on emergency obstetric and newborn care assessment 2016 data. BMC Health Serv Res. 2019;19(1):1014.

11. Chekol BM, Abdi DA, Adal TA. Dimensions of patient satisfaction with comprehensive abortion care in Addis Ababa, Ethiopia. Reproductive health. 2016;13(1):144.

12. Alemayehu B, et al. Magnitude and associated factors of repeat induced abortion among reproductive age group women who seeks abortion Care Services at Marie Stopes International Ethiopia Clinics in Addis Ababa, Ethiopia. Reproductive health. 2019;16(1):76.

13. Worku SA, Ahmed SM, Mulushewa TF, Unmet need for family planning and its associated factor among women of reproductive age in Debre Berhan Town, Amhara, Ethiopia. BMC research notes, 2019. 12(1): p. 143.

14. Moore AM, et al. The estimated incidence of induced abortion in Ethiopia, 2014: changes in the provision of services since 2008. International perspectives on sexual reproductive health. 2016;42(3):111.

15. Mitiku S, et al., Prevalence of Induced Abortion and Associated Factors among Wachamo University Regular Female Students, Southern Ethiopia. Journal of Pharmacy and Alternative Medicine, 2015.

16. Pourhoseingholi $M$, et al. Alternative for the cox regression model: using parametric models to analyze the survival of cancer patients. Iranian Journal of Cancer Prevention. 2011;4(1):1-9.

\section{Figures}
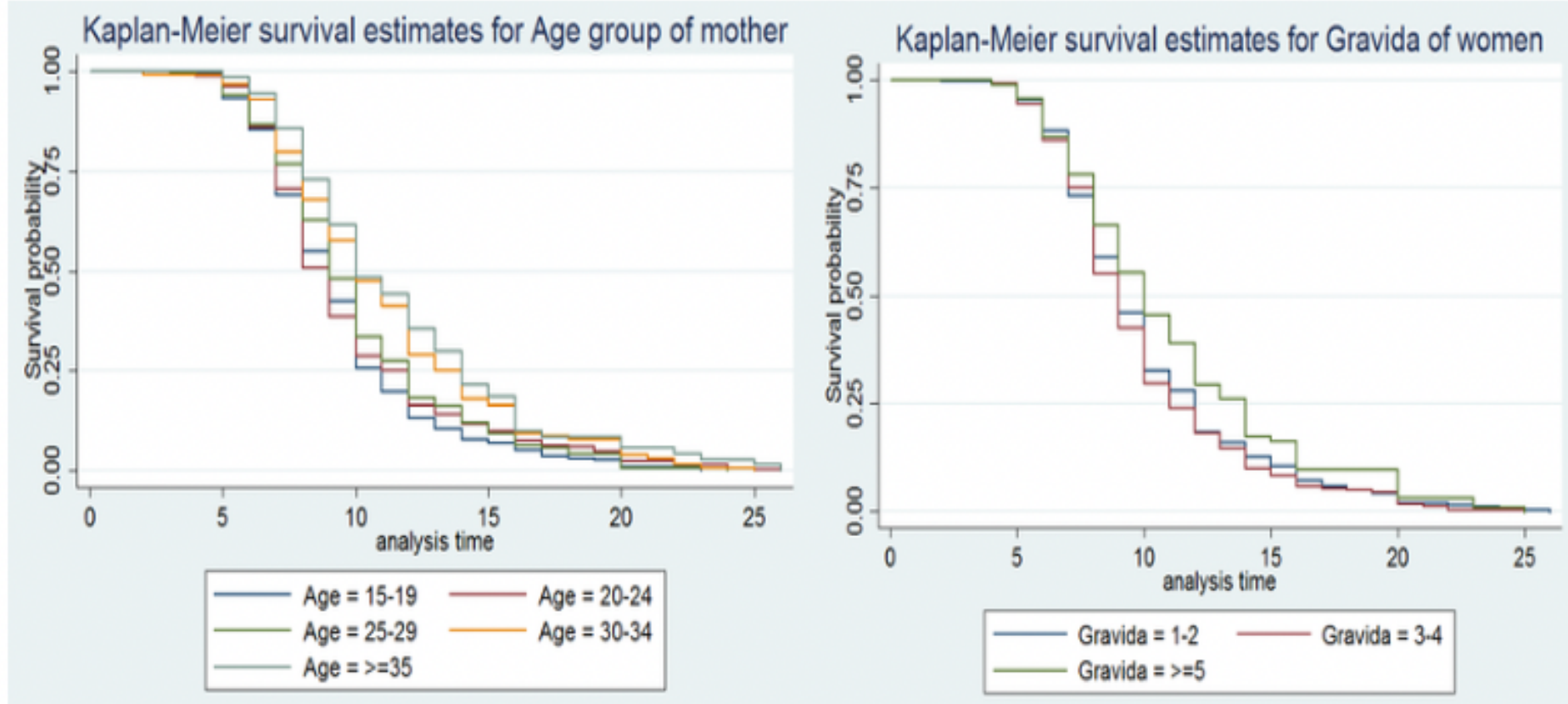
Figure 1

KM survival time plot by Age of mother and Gravida of first trimester abortion for women
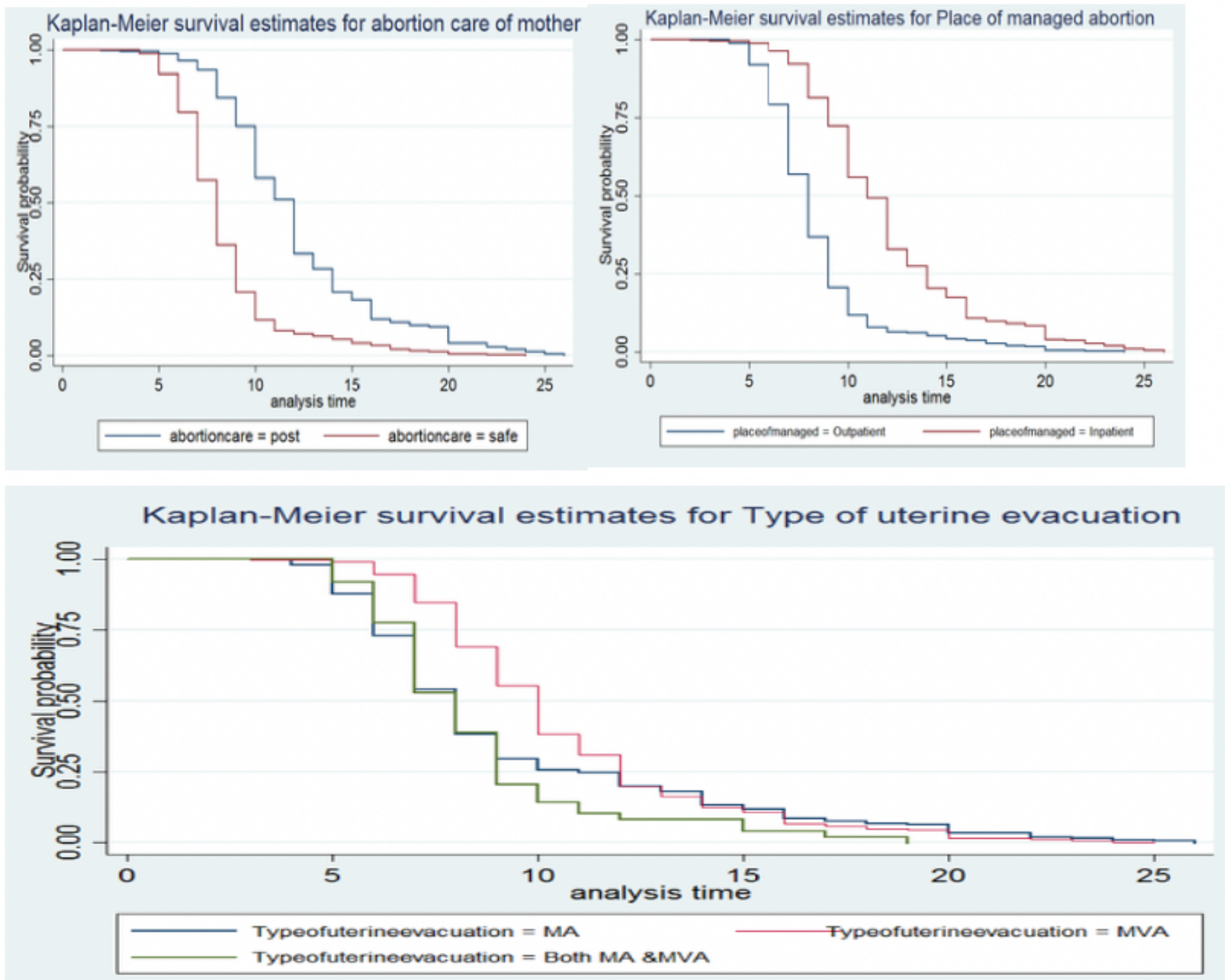

Figure 2

Kaplan-Meier survival time plot by abortion care, place of managed and type of uterine evacuation of first trimester abortion for women 

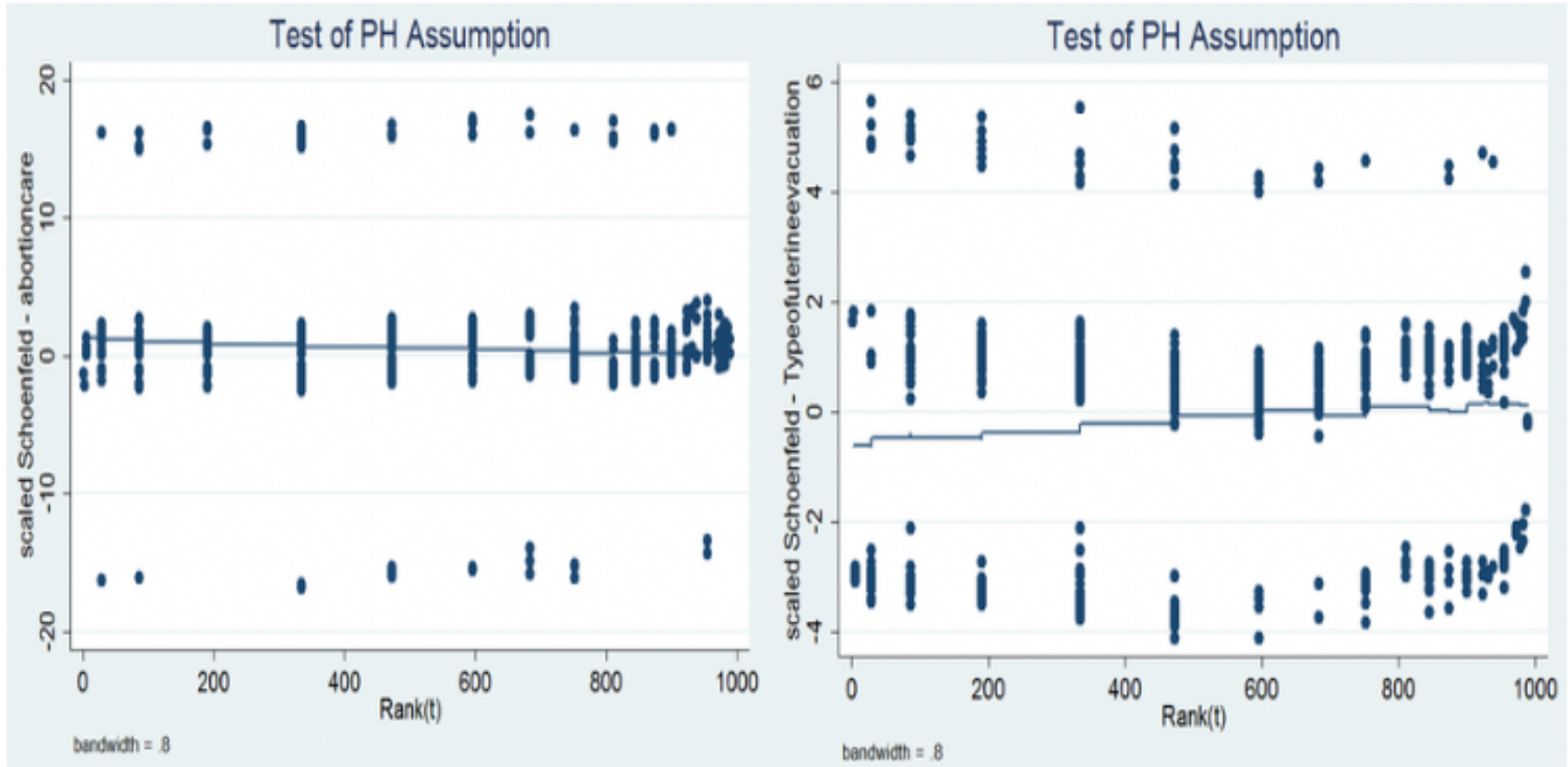

Figure 3

The plot of Scaled Schoenfeld residuals for abortion care and Type of uterine evacuation to check PH assumption 

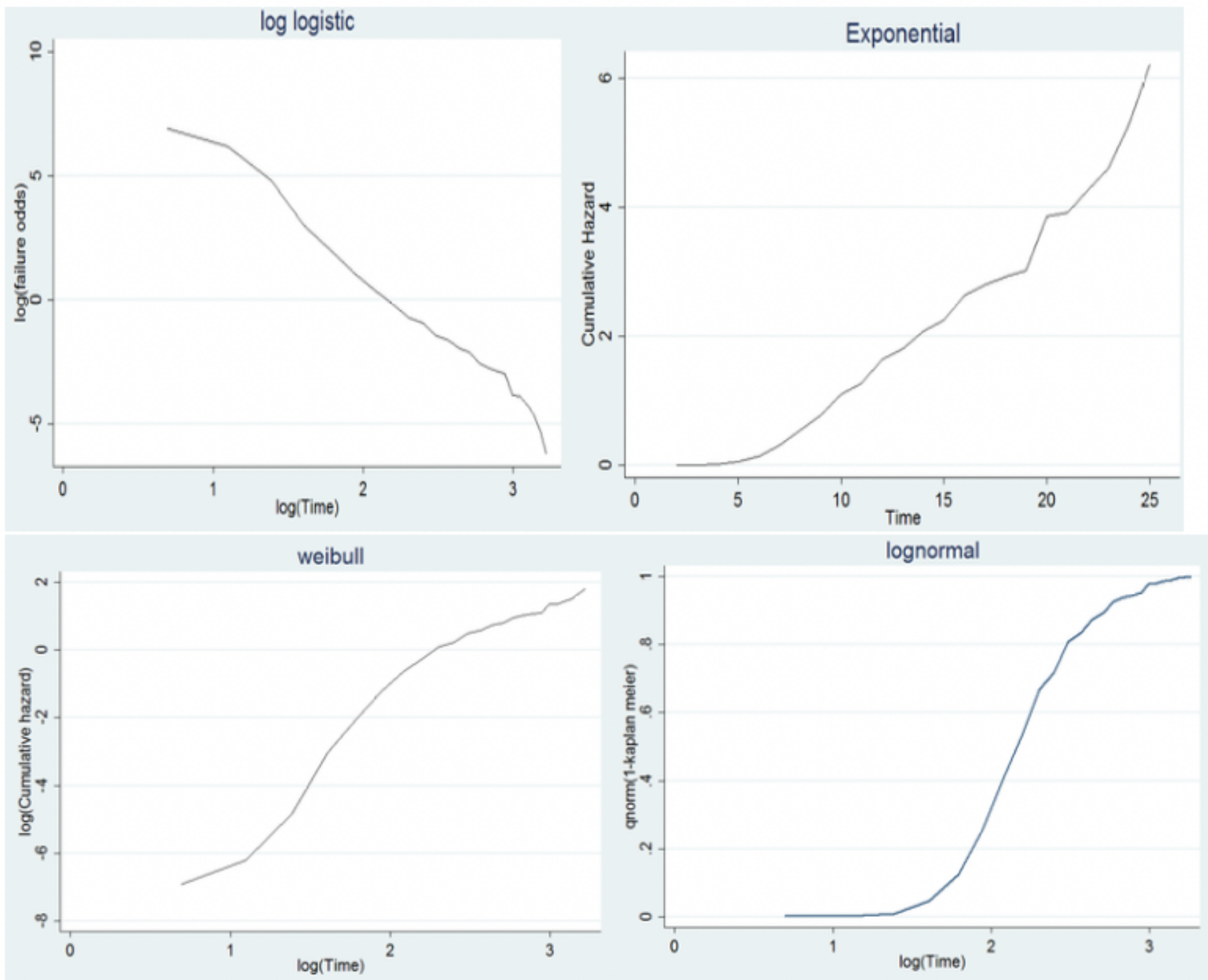

Figure 4

Graphs of Log-logistic, Exponential, Weibull and Log-normal baseline distributions for survival time of women's abortion data.

\section{Supplementary Files}

This is a list of supplementary files associated with this preprint. Click to download.

- Supplementarymaterial1.docx 